\title{
Serratia marcescens Causing Pneumonia - A Rare Case Report
}

Rajashree Panigrahy*

IMS \& SUM Hospital, Bhubaneswar, Khurda, India

\begin{abstract}
Serratia marcescens is an opportunistic, gram negative, nosocomial pathogen which belongs to family, Enterobacteriaceae. It was originally considered to be an innocuous, non-pathogenic, saprophytic water organism. The main risk factors for bacteraemia/sepsis which is caused by Serratia is hospitalization, placement of intravenous catheters, intraperitoneal catheters and urinary catheters and prior instrumentation of the respiratory tract. An important characteristic is its ability to produce a beta-lactamase which confers resistance to the broad spectrum beta lactam antibiotics, which often complicates the therapy. Here we present a case of pneumonia caused by Serratia marcescens in a 3 year old child, without having any significant predisposing factors.
\end{abstract}

Keywords: Serratia marcescens; Pneumonia; Beta lactamase

\section{Introduction}

Serratia marcescens is an opportunistic, gram negative, nosocomial pathogen which belongs to family, Enterobacteriaceae. It was originally considered to be an innocuous, non-pathogenic, saprophytic water organism and it was often used as a biological marker because of its easily recognizable red colonies [1]. Intensive care units are often involved in the epidemics of the colonization and the infection with Serratia marcescens. The important reservoirs in epidemics are the digestive tract, the respiratory tract, the urinary tracts and the perineum of neonates and the artificial nails of adults and health care workers. Studies show Serratia marcescens accounts for only $1-2 \%$ of the nosocomial infections which are mostly confined to the respiratory tract, the urinary tract, surgical wounds and soft tissues. In UTI, $30-35 \%$ of the patients are asymptomatic and most of the patients have a history of instrumentation. The mortality rate is very high in the nosocomial blood stream infections and in meningitis and endocarditis. The main risk factors for bacteraemia/sepsis which is caused by Serratia is hospitalization, placement of intravenous catheters, intraperitoneal catheters and urinary catheters and prior instrumentation of the respiratory tract [2-5]. An important characteristic is its ability to produce a beta lactamase which confers resistance to the broad spectrum, beta lactum antibiotics, which often complicates the therapy. Serratia marcescens causes both opportunistic and nosocomial infections. The major factors that are involved in the development of the Serratia infection include contamination of the respiratory equipment and poor catheterization techniques. But in our case, there is no history of hospitalisation or instrumentation or compromising immunity history.

\section{Case History}

A 3 years old male child presented to the outpatient department of Paediatrics with history of cough and cold for 10 days which was associated with high grade fever and intermittent vomiting. He had received a injectable antibiotic and supportive therapy at local hospital for the same and referred here. On presentation, child was febrile (temp $=102.3 \mathrm{~F}$ ), sick looking with tachycardia (Heart Rate=166/min), $\mathrm{SPO}_{2}$ (Oxygen saturation $)=82 \%$ with air, tachypnea (Respiratory Rate $=72 / \mathrm{min}$ ) with features of respiratory distress. On examination, no pallor, no cyanosis, bilateral chest crepitation and ronchi with liver $4 \mathrm{~cm}$ palpable below right subcostal margin.

Arterial Blood Gas analysis shows respiratory alkalosis. $(\mathrm{pH}=7.54$, $\left.\mathrm{pCO}_{2}=30 \mathrm{~mm} \mathrm{Hg}\right)$ with hypoxemia $\left(\mathrm{pO}_{2}=50 \%\right)$. Total Leucocyte Count is $20,400 / \mathrm{mm}^{3}$ with neutrophilia (76\%) and chest X-ray shows bilateral non-homogenous opacity. Child was ventilated and blood and broncho-alveolar lavage (BAL) was sent for culture and sensitivity.
Child started with broad spectrum antibiotic, ceftriaxone and vancomycin. Blood culture showed no growth but BAL showed growth of Serratia marcescens (identified by conventional biochemical tests and confirmed by VITEK 2 system). Isolate was found to be susceptible to amikacin, netilmycin, tobramycin, and imipenem. On the day of isolation of Serratia marcescens, again BAL sample was collected and treated with imipenem. Again Serratia marcescens with same antibiotic sensitivity pattern was isolated in pure culture from the BAL sample. Within 3 days of strating imipenem, the child was symptom free. The chest $\mathrm{x}$-ray was normal at the time of discharge from the hospital (after 7 days).

Because no other causative agent was found, the pneumonia described here seems to have been caused by the isolated organism Serratia marcescens.

\section{Discussion and Conclusion}

Although S. marcescens was considered to be an innocuous, nonpathogenic organism, over the last few decades, they have become an opportunist pathogen causing nosocomial infections. Reports have shown rare cases of $S$. marcescens in nonhospital settings which are linked to patients with immune deficiencies or chronic debilitating diseases. S. marcescens are also capable of causing diseases in a diverse group of organisms including animals, coral, insects, and plants [6,7].

But in our case it is the primary cause of pneumonia in the child. It may come from the soil, result of unhygienic habits, as the patient belongs to a low socio-economic status group.

The contributory factors for the spread of the hospital acquired infections include inadequate infection control practices which are coupled with the growing range of the modern medical procedures. The soap dispenser which was infected with Serratia was removed from the Intensive Care Unit (ICU) and this probably helped in limiting the spread of the infection [8]. A similar spread of the infection through soap dispensers, which was caused by Serratia marcescens in hospitalized patients as a result of inadequate hand hygiene, has been reported by others also $[9,10]$. Alcohol based hand antiseptic were able to remove pathogens from $80 \%$ of the health care workers [11].

*Corresponding author: Rajashree Panigrahy, Associate Professor, IMS \& SUM Hospital, Microbiology, Kalinga Nagar, Khandagiri, Bhubaneswar, Khurda-751003, India, Tel: 09437216735; E-mail: dr.rajashreep@gmail.com

Received February 27, 2015; Accepted April 07, 2015; Published April 11, 2015

Citation: Panigrahy R (2015) Serratia marcescens Causing Pneumonia - A Rare Case Report. J Pulm Respir Med 5: 254. doi:10.4172/2161-105X.1000254

Copyright: () 2015 Panigrahy R. This is an open-access article distributed under the terms of the Creative Commons Attribution License, which permits unrestricted use, distribution, and reproduction in any medium, provided the original author and source are credited. 
But no such associated factors are found in this case. No other Serratia group infection was noted in the same ward or the entire hospital during this period.

Troillet et al. reported imipenem resistance in $11 \%$ of the clinical isolates of Serratia species [12]. Another study showed that the Serratia marcescens isolates were sensitive to imipenem, but the resistance to meropenem was high [13]. Kumar and Worobee studied fluoroquinolone resistance of $S$. marcescens. Our isolate also resistant to flouroquinolones, cephalosporins, penicillin group, and sensitive to aminoglycosides and imipenem.

Although S. marcescens is an opportunistic pathogen causing a plethora of nosocomial infections in humans and some cases have been reported in animals and many strains have become resistant to a variety of drugs but clinical isolates should not be ignored. There pathogenicity should be proved and appropriate treatment should be given to the patients.

\section{References}

1. Merlino CP (1924) Bartolomeo Bizio's Letter to the most Eminent Priest, Angelo Bellani, Concerning the Phenomenon of the Red Colored Polenta. J Bacteriol 9: 527-543.

2. Yoon HJ, Choi JY, Park YS, Kim CO, Kim JM, et al. (2005) Outbreaks of Serratia marcescens bacteriuria in a neurosurgical intensive care unit of a tertiary care teaching hospital: a clinical, epidemiologic, and laboratory perspective. Am J Infect Control 33: 595-601.

3. Casolari C, Pecorari M, Fabio G, Cattani S, Venturelli C, et al. (2005) A simultaneous outbreak of Serratia marcescens and Klebsiella pneumoniae in a neonatal intensive care unit. J Hosp Infect 61: 312-320.
4. Greco Stewart VS, Brown EE, Parr C, Kalab M, Jacobs MR, et al. (2012) Serratia marcescens strains implicated in adverse transfusion reaction form biofilms in platelet concentrates and dem?onstrate reduced detection by automated cultures. Vox Sang. 2012 April; 102(3): 212-20.

5. Crivaro V, Bagattini M, Salza MF, Raimondi F, Rossano F, et al. (2007) Risk factors for extended-spectrum beta-lactamase-producing Serratia marcescens and Klebsiella pneumoniae acquisition in a neonatal intensive care unit. J Hosp Infect 67: 135-141.

6. Coulthurst SJ, Williamson NR, Harris AK, Spring DR, Salmond GP (2006) Metabolic and regulatory engineering of Serratia marcescens: mimicking phage-mediated horizontal acquisition of antibiotic biosynthesis and quorumsensing capacities. Microbiology 152: 1899-1911.

7. Hejazi A, Falkiner FR (1997) Serratia marcescens. J Med Microbiol 46: 903-912.

8. Khanna A, Khanna M, Aggarwal A (2013) Serratia marcescens- a rare opportunistic nosocomial pathogen and measures to limit its spread in hospitalized patients. J Clin Diagn Res 7: 243-246.

9. Buffet-Bataillon S, Rabier V, Bétrémieux P, Beuchée A, Bauer M, et al. (2009) Outbreak of Serratia marcescens in a neonatal intensive care unit: contaminated unmedicated liquid soap and risk factors. J Hosp Infect 72: 17-22.

10. Rabier V, Bataillon S, Jolivet-Gougeon A, Chapplain JM, Beuchée A, et al (2008) Hand washing soap as a source of neonatal Serratia marcescens outbreak. Acta Paediatr 97: 1381-1385.

11. McNeil SA, Foster CL, Hedderwick SA, Kauffman CA (2001) Effect of hand cleansing with antimicrobial soap or alcohol-based gel on microbial colonization of artificial fingernails worn by health care workers. Clin Infect Dis 32: 367-372.

12. Troillet N, Carmeli Y, Venkataraman L, DeGirolami P, Samore MH (1999) Epidemiological analysis of imipenem-resistant Serratia marcescens in hospitalized patients. J Hosp Infect 42: 37-43.

13. Lefort A, Righi S, Jauréguy $F$, Bégué T, Robineau M, et al. (2005) Serratia marcescens prosthesis infection successfully treated with meropenem after imipenem failure. J Infect 51: E45-47. 